\title{
Effet cosinus sur un modèle visqueux de type Saint-Venant et ses équations limites de type quasi-géostrophique et lacs
}

\author{
Carine Lucas ${ }^{\text {a }}$ \\ a Université Joseph Fourier, INRIA, LJK UMR5224, 51 Rue des Mathématiques, B.P. 53 , 38041 Grenoble, France \\ Reçu le ${ }^{* * * * *} ;$ accepté après révision le +++++ \\ Présenté par
}

\begin{abstract}
Résumé
L'ordre d'approximation nécessaire à l'obtention d'un modèle visqueux de type Saint-Venant avec force de Coriolis, à partir des équations de Navier-Stokes à surface libre, dépend du lien entre le nombre de Reynolds et le rapport d'aspect. Avec une méthodologie certes classique dans le domaine de l'analyse asymptotique (développement en série), nous montrerons que de nouveaux termes dépendant du cosinus de la latitude ont été oubliés dans des papiers récents et doivent être pris en compte pour certaines applications en géophysique. En revanche, lors de l'étude des films minces en rotation, ces termes n'apparaissent pas. Nous donnerons également les limites de type quasi-géostrophique et lacs correspondant aux équations avec effet cosinus. Tous ces modèles sont bien posés (existence de solutions faibles globales) et nous montrons que ces termes en cosinus modifient les ondes équatoriales. Pour citer cet article : C.Lucas, C. R. Acad. Sci. Paris
\end{abstract}

\section{Abstract}

Cosine effect on a viscous Shallow Water model and its quasigeostrophic and lake equations limits. To get a viscous Shallow Water type model with Coriolis force from free surface Navier-Stokes equations, the required order of approximation depends on the relative order between the Reynolds number and the aspect ratio. Even if the methodology is classical in the field of asymptotic analysis (ansatz), we'll prove that new terms depending on the latitude cosine have been omitted in recent papers and must be taken into account for some applications in geophysics. However, these terms do not appear when we study rotating thin films. We'll also give the quasi-geostrophic and the lake limits corresponding to the equations with cosine effect. All these models are well posed (existence of global weak solutions) and we show that the cosine terms affect equatorial waves. To cite this article: C. Lucas, C. R. Acad. Sci. Paris

Email address: Carine.Lucas@imag.fr (Carine Lucas). 


\section{Abridged English version}

The required order of approximation to get a viscous Shallow Water type model with Coriolis force from the incompressible free surface Navier-Stokes equations depends on the link between the Reynolds number Re and the aspect ratio $\epsilon$. The Reynolds number quantifies the relative importance of inertial and viscous forces, and $\epsilon$ is the ratio between the height and the length of the domain. According to the applications we aim at, for example flows in great lakes, in seas and oceans, or spreading of a rotating thin film, cosine effect should be taken into account or not. For the reader's convenience, we can get an idea of the significance of terms calculating characteristic scales in some particular cases.

Flows in a great lake such as Ontario lake $(H \approx 200 \mathrm{~m}, L \approx 300 \mathrm{~km})$ or in the north Atlantic $(H \approx 7000 \mathrm{~m}, L \approx 9000 \mathrm{~km})$ with a mean horizontal velocity $u_{\text {car }}$ of order $10^{-2} \mathrm{~m} \cdot \mathrm{s}^{-1}$ give, considering a turbulent viscosity, an aspect ratio of order $10^{-3}$ and a Reynolds number of order $10^{3}$. The flow is inviscid at the main order ( $c f .[11])$ and with the sine part of the Coriolis term. If we wish to parametrize the viscous effects, we'll prove that the cosine part of the Coriolis force must be taken into account. Next, we give the quasi-geostrophic and the lake limits corresponding to this viscous model with cosine effect. By this way, we generalize existing geophysical models.

The spreading of a rotating thin film such as in centrifugation process $\left(H \approx 10^{-4} \mathrm{~m}, L \approx 10^{-2} \mathrm{~m}\right)$ gives an aspect ratio of order $10^{-2}$ and can be contemplated whether in a laminar or in a turbulent regime. In the laminar case, the Reynolds number can be considered as fixed. The flow is then viscous at main order ( $c f .[12])$ and only takes into account the sine part of the Coriolis term: viscosity and cosine effect are not coupled anymore.

The methodology used to get formally the Shallow Water equations is classical, the difficulty being that the nonlinear terms depend on the bottom conditions we're studying ( $c f$. . [13]), and on the relative order between the Reynolds number and the aspect ratio. Here, we'll consider Navier type boundary conditions as in [5]; see figure 1 for explicit equations and boundary conditions.

Depending on the applications we consider, namely geophysical flows or thin films, we find two different viscous Shallow-Water systems:

Applications in geophysics. When $1 / \operatorname{Re}=O(\epsilon)$, the viscous Shallow Water system is

$$
\begin{aligned}
& \partial_{t} h+\operatorname{div}(h u)=0, \\
& \partial_{t}(h u)+\operatorname{div}(h u \otimes u)-\frac{2}{\operatorname{Re}} \operatorname{div}(h D(u))-\frac{2}{\operatorname{Re}} \nabla(h \operatorname{div} u)+\frac{1}{\mathrm{Fr}^{2}} h \nabla(h+b)+\alpha_{0}(h) u \\
& \quad+\frac{1}{\operatorname{Ro}}\left(\sin \theta h u^{\perp}-\epsilon \frac{\cos \theta}{2} h^{2} \operatorname{div} u e_{1}-\epsilon \frac{\cos \theta}{2} \nabla\left(h^{2} u_{1}\right)\right)+\frac{\epsilon \cos \theta}{\operatorname{Ro}}\left(h u \cdot \nabla b e_{1}-u_{1} h \nabla b\right)=0,
\end{aligned}
$$

where $D(u)=\left(\nabla u+{ }^{t} \nabla u\right) / 2, \alpha_{0}(h)=r_{0} /\left(\epsilon\left(1+\epsilon \operatorname{Re} r_{0} h / 3\right)\right)$, Ro is the Rossby number and Fr the Froude number. In this equation, $u$ is the vertical average of the horizontal component of the velocity at order $\epsilon$. The cosine effect, that is the fact that some terms depend on $\cos \theta$, seems new for the author. Such a model allows to generalize usual viscous geophysical models such as the quasi-geostrophic or lake systems with Coriolis force, see respectively equations (10) and (11)-(12). We prove that our models are well posed when we take into account capillary effects that is when we add the term $-\sigma h \nabla \Delta h$ to the momentum equations and when we neglect the term $\nabla(h \operatorname{div} u)$. We also show that the cosine terms change the speed of equatorial waves.

Applications for thin films. If $1 / \operatorname{Re}=O(1)$, we directly get at the main order

$$
\begin{aligned}
& \partial_{t} h+\operatorname{div}(h u)=0, \\
& \partial_{t}(h u)+\operatorname{div}(h u \otimes u)-\frac{2}{\operatorname{Re}} \operatorname{div}(h D(u))-\frac{2}{\operatorname{Re}} \nabla(h \operatorname{div} u)+\frac{1}{\mathrm{Fr}^{2}} h \nabla(h+b)+r_{0} u+\frac{1}{\operatorname{Ro}} \sin \theta h u^{\perp}=0 .
\end{aligned}
$$


The limit model is also viscous, but the friction terms are differents, as well as the Coriolis term (no cosine effect). This asymptotic corresponds to the dynamics of a rotating thin film in laminar regime.

\section{Introduction}

Pour obtenir un modèle visqueux de type Saint-Venant avec force de Coriolis, à partir des équations de Navier-Stokes incompressibles à surface libre, l'ordre d'approximation doit être soigneusement choisi en fonction du lien entre le nombre de Reynolds et le rapport d'aspect. Suivant les applications visées, par exemple l'écoulement dans les grands lacs, dans les mers et océans, ou l'étalement d'un film mince en rotation, l'effet cosinus devra ou non être pris en compte. On peut se donner une idée de l'importance des termes en calculant les échelles caractéristiques dans certains cas particuliers. On renvoie pour cela le lecteur à la version abrégée en anglais.

La méthodologie utilisée pour obtenir formellement les équations de Saint-Venant est classique mais la gestion des termes non linéaires dépend des conditions au fond étudiées ( $c f$. [13], [8]). Nous considérerons ici des conditions au fond de type Navier. Suivant les applications considérées, nous pouvons obtenir différents modèles visqueux. En ce qui concerne les modèles géophysiques pour lesquels le nombre de Reynolds est assez grand, inversement proportionnel au rapport d'aspect, le système de Saint-Venant visqueux s'écrit (1)-(2). Pour ce qui est des écoulements de films minces où la viscosité est alors d'ordre un, on obtient le système (3)-(4).

Dans toute la suite, nous nous focaliserons sur l'aspect géophysique avec un nombre de Reynolds inversement proportionnel au rapport d'aspect. Nous obtiendrons un modèle avec des termes nouveaux qui peuvent être importants proche de l'équateur et qu'il faudrait prendre en compte par exemple dans [4] car ils changent les vitesses des ondes équatoriales.

\section{Obtention du nouveau modèle à partir de Navier-Stokes visqueux}

Les équations de Saint-Venant, pour un fluide newtonien, s'obtiennent à partir des équations de NavierStokes à surface libre (voir figure 1).

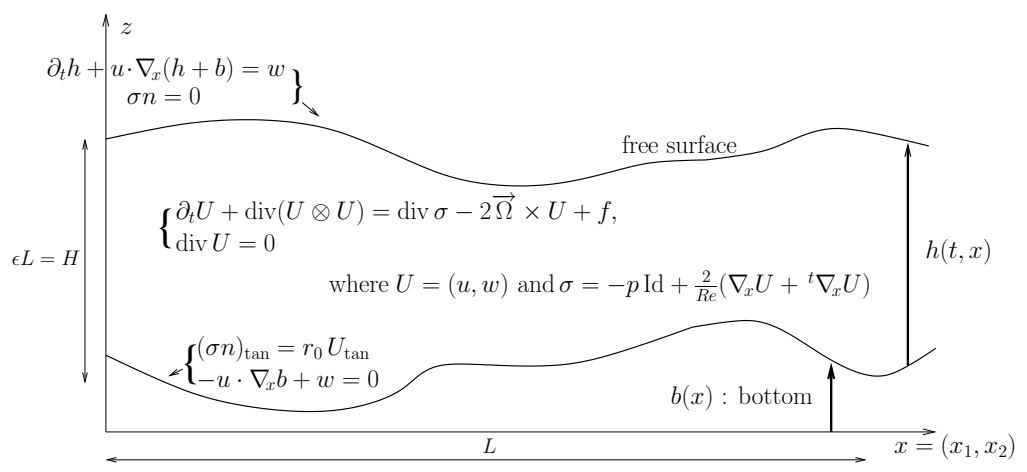

FIG. 1. Domaine et conditions au bord

On rappelle que $\vec{\Omega}=\Omega(0, \cos \theta, \sin \theta)$, où $\Omega\left(=7.310^{-5} s^{-1}\right)$ est la fréquence de rotation de la Terre et $\theta$ la latitude, représente le vecteur rotation de la Terre. Lors de la mise sous forme non-dimensionnelle, on choisit un nombre de Rossby égal à Ro $=u_{\text {car }} / 2 L \Omega$ où $u_{\text {car }}$ est l'échelle caractéristique de la vitesse 
horizontale, et Fr le nombre de Froude habituel. On réalise un développement asymptotique suivant le rapport d'aspect $\epsilon=H / L$ supposé petit : $U=U^{0}+\epsilon U^{1}+\ldots$ et $h=h^{0}+\epsilon h^{1}+\ldots$ avec $U=(u, w)$, où $u=\left(u_{1}, u_{2}\right)$ désigne la composante horizontale du champ de vitesse. Les modèles obtenus vont alors dépendre de l'ordre, par rapport à ce petit paramètre $\epsilon$, des coefficients physiques mis en jeu. Au fond, en variables non-dimensionnelles, la condition de Navier est du type $(\sigma n)_{\tan }=r_{0} u$ où $r_{0}$ est d'ordre $\epsilon$. Par la suite, on notera $u$ la moyenne verticale de la composante horizontale de la vitesse $u^{0}+\epsilon u^{1}$.

La contrainte de divergence nulle moyennée en verticale donne de manière standard l'équation de la surface libre $\partial_{t} h+\operatorname{div}(h u)=0$. A partir de la composante verticale des moments, on trouve, à l'ordre $\epsilon$, l'approximation hydrostatique suivante :

$$
p(t, x, z)=\frac{1}{\operatorname{Fr}^{2}}(h-z)-\frac{1}{\operatorname{Re}}(\operatorname{div} u)_{\left.\right|_{z=h+b}}-\frac{1}{\operatorname{Re}} \operatorname{div} u+\epsilon \frac{\cos \theta}{\operatorname{Ro}} \int_{h}^{z} u_{1} .
$$

On utilise ensuite la composante horizontale des moments et les conditions au fond et en surface à l'ordre principal pour obtenir

$$
\partial_{z}^{2} u=O(\epsilon), \quad \partial_{z} u_{\left.\right|_{z=b}}=O(\epsilon), \quad \partial_{z} u_{\left.\right|_{z=h+b}}=O(\epsilon)
$$

donc, au premier ordre, $u$ ne dépend pas de $z: u^{0}(t, x, z)=u^{0}(t, x)$, qui reste inconnu. La dynamique de $u^{0}$ et $h^{0}$ est obtenue en moyennant sur la verticale la composante horizontale des moments à l'ordre 1 . Elle est gouvernée par le système de Saint-Venant hyperbolique classique (cf. [11]) suivant

$$
\begin{aligned}
& \partial_{t} h^{0}+\operatorname{div}\left(h^{0} u^{0}\right)=0, \\
& \partial_{t}\left(h^{0} u^{0}\right)+\operatorname{div}\left(h^{0} u^{0} \otimes u^{0}\right)+\frac{1}{\operatorname{Fr}^{2}} h^{0} \nabla\left(h^{0}+b\right)+r_{0} u^{0}+\frac{1}{\operatorname{Ro}_{0}} \sin \theta h^{0}\left(u^{0}\right)^{\perp}=0 .
\end{aligned}
$$

Si l'on désire avoir un système visqueux, paramétrant par exemple les effets de petites échelles, on itère à l'ordre suivant. On doit alors garder le terme en $\epsilon \cos \theta w e_{1}$ de l'équation des moments : il donne les termes en $e_{1}$ dans (2), les autres termes en cosinus provenant de (5). Le système obtenu (1)-(2) nous montre que viscosité et effet cosinus sont étroitement liés.

Si l'on considère le nombre de Reynolds d'ordre 1, en suivant le même procédé que précédemment, on obtient à l'ordre 1 le système (3)-(4) au lieu de (6)-(7) : l'analyse asymptotique à l'ordre 1 suffit pour voir la trace de la viscosité au sein de l'équation de Saint-Venant.

\section{Quelques résultats mathématiques}

A la connaissance de l'auteur, le modèle de Saint-Venant visqueux avec force de Coriolis complète semble nouveau. Nous nous intéressons alors en connaître mieux les propriétés mathématiques en y incluant pour plus de généralité les termes de tension de surface. Considérons donc le modèle suivant

$$
\begin{aligned}
& \partial_{t} h+\operatorname{div}(h u)=0, \\
& \partial_{t}(h u)+\operatorname{div}(h u \otimes u)-\frac{2}{\operatorname{Re}} \operatorname{div}(h D(u))+\frac{1}{\operatorname{Fr}^{2}} h \nabla(h+b)+\alpha_{0}(h) u-\sigma h \nabla \Delta(h+b) \\
& \quad+\frac{1}{\operatorname{Ro}}\left(\sin \theta h u^{\perp}-\epsilon \frac{\cos \theta}{2} h^{2} \operatorname{div} u e_{1}-\epsilon \frac{\cos \theta}{2} \nabla\left(h^{2} u_{1}\right)\right)+\frac{\epsilon \cos \theta}{\operatorname{Ro}}\left(h u \cdot \nabla b e_{1}-u_{1} h \nabla b\right)=0, \\
& \left.h\right|_{t=0}=h_{0},\left.\quad(h u)\right|_{t=0}=m_{0} .
\end{aligned}
$$

Soit $\mathcal{D}$ un domaine périodique; on a les résultats suivants : 
Théorème 3.1 Soit $h_{0} \in L^{2}(\mathcal{D})$ avec $h_{0} \geq 0, m_{0} / \sqrt{h}_{0} \in L^{2}(\mathcal{D})$ avec $m_{0} / \sqrt{h}_{0}=0$ quand $h_{0}=0$ et $\log \min \left(h_{0}, 1\right) \in L^{1}(\mathcal{D})$. Supposons que $b \in H^{3}(\mathcal{D})$ tel que $b \geq c>0$ et $\alpha_{0}>0, \sigma>0$, il existe alors une solution globale faible $(h, u)$ de (8)-(9). Considérant $\operatorname{Ro}=\eta, \mathrm{Fr}^{2}=F \eta^{2}, b=\eta \tilde{b}$ on montre alors quand $\eta$ tend vers 0 la convergence d'une sous-suite vers une solution globale faible de (10). Si $\mathrm{Fr}^{2}=\delta$ avec Ro et $\epsilon$ fixés, on obtient également la convergence vers une solution globale faible de (11)-(12).

Nous montrons également que la dynamique des ondes équatoriales est affectée par cet effet cosinus pour un nombre de Rossby de l'ordre du rapport d'aspect. Nous allons expliquer rapidement par la suite les diverses asymptotiques et les étapes de démonstrations du théorème.

\section{4. Équations quasi-geostrophiques et équations des lacs}

Nous voulons maintenant voir l'influence de ces nouveaux termes en cosinus sur les modèles habituellement utilisés en géophysique et plus précisément sur les équations quasi-géostrophiques et les équations des lacs avec viscosité.

Équations quasi-géostrophiques. Les équations quasi-géostrophiques sont très largement utilisées pour la modélisation et la prévision de la circulation océanique et atmosphérique aux latitudes moyennes. Pour les obtenir, on suppose que $\operatorname{Ro}=\eta, \mathrm{Fr}^{2}=F \eta^{2}, b=\eta \tilde{b}$ et que $\epsilon$ est fixé. On développe alors les variables : $u=u^{0}+\eta u^{1}+\cdots, h=1+F \eta h^{1}+\cdots$.

Grâce à l'équation (1), nous pouvons affirmer qu'il existe une fonction de courant $\psi$ telle que $u^{0}=\nabla_{x}^{\perp} \psi$. L'équation (2) quant à elle s'écrit au premier ordre $h^{1}=\left(\sin \theta-\frac{\epsilon}{2} \cos \theta \partial_{x_{2}}\right) \psi-\frac{\tilde{b}}{F}$, et au second ordre nous donne l'équation quasi-géostrophique :

$$
\left(\partial_{t}+u \cdot \nabla\right)\left(\left(\partial_{x_{1}}^{2}+\left(1+\frac{F \epsilon^{2}}{4} \cos ^{2} \theta\right) \partial_{x_{2}}^{2}\right) \psi-F \sin ^{2} \theta \psi+\left(\sin \theta-\frac{\epsilon}{2} \cos \theta \partial_{x_{2}}\right) \tilde{b}\right)=\frac{\Delta^{2} \psi}{\operatorname{Re}}-\alpha_{0}(1) \Delta \psi \cdot(10)
$$

Notons que le modèle obtenu présente une certaine anisotropie en la vorticité transportée. Les différents exemples numériques proposés dans [9] montrent que l'effet cosinus peut avoir une grande influence en temps long.

Équations des lacs. Les équations des lacs sont obtenues comme la limite faible profondeur des équations d'Euler ou de Navier-Stokes à trois dimensions, avec l'hypothèse du toit rigide, dans un bassin horizontal avec une topographie variable. On suppose donc que $\mathrm{Fr}^{2}=\delta$ avec Ro et $\epsilon$ fixés. On écrit encore un développement asymptotique des variables : $u=u^{0}+\delta u^{1}+\ldots, h=h^{0}+\delta h^{1}+\ldots$. Au premier ordre, l'équation (2) permet d'affirmer que $h^{0}+b$ est une constante en espace et en temps, donnée par les valeurs initiales (on peut supposer que cette constante est égale à 1 ). Ensuite, si on note $h_{b}=1-b$, avec le second ordre et l'équation (1) on obtient :

$$
\begin{aligned}
& \operatorname{div}\left(h_{b} u\right)=0, \\
& \begin{array}{r}
\partial_{t}\left(h_{b} u\right)+\operatorname{div}\left(h_{b} u \otimes u\right)-\frac{2}{\operatorname{Re}} \operatorname{div}\left(h_{b} D(u)\right)-\frac{2}{\operatorname{Re}} \nabla\left(h_{b} \operatorname{div} u\right)+h_{b} \nabla p \\
+\alpha_{0}\left(h_{b}\right) u+\frac{h_{b}}{\operatorname{Ro}}\left(\sin \theta-\frac{\epsilon}{2} \cos \theta h_{b} \partial_{x_{2}}\right) u^{\perp}=0 .
\end{array}
\end{aligned}
$$

Notre étude permet d'inclure les termes de Coriolis dans le modèle visqueux établi dans [6]. Notons que l'on peut écrire son analogue sans viscosité. On obtient alors une généralisation du modèle des lacs étudié par exemple dans [3]. 


\section{Points clefs sur l'étude mathématique des modèles obtenus}

Montrer l'existence globale de solutions faibles pour des modèles de Saint-Venant visqueux dégénérés sans termes de Coriolis en cosinus et sans le terme $\nabla(h \operatorname{div} u)$ est longtemps resté un problème ouvert (cf. [7]). La principale difficulté a été surmontée récemment dans [1] grâce à une nouvelle entropie (BD entropie) qui implique que le gradient de $\sqrt{h}$ reste borné pour tout temps s'il l'est à l'instant initial. Nous montrons que même si les termes en cosinus sont d'ordre $\epsilon$, la nouvelle entropie ne semble pouvoir être satisfaite que si l'on prend en compte les effets de capillarité qui sont du même ordre que le nombre de Reynolds pour des grandes étendues d'eau. Nous démontrons ensuite rigoureusement la convergence des équations de Saint-Venant vers l'équation quasi-géostrophique et l'équation des lacs avec termes en cosinus en nous inspirant de [1] et [2].

On peut également étudier les ondes équatoriales du modèle de Saint-Venant visqueux afin de mieux comprendre l'effet cosinus dans une zone où il devrait jouer un rôle important. Le lecteur intéressé par une étude mathématique de type fluides tournants pour Saint-Venant visqueux avec terme en sinus $\left(\beta x_{2}\right)$ est renvoyé à [4]. La prise en compte de l'effet cosinus est nécessaire car dans ce domaine d'application $\epsilon \approx$ Ro; le calcul formel des vitesse d'ondes de Kelvin donne en effet :

$$
c_{1}=\sqrt{\frac{h}{\mathrm{Fr}^{2}}+\epsilon^{2} \frac{h^{2}}{(2 \mathrm{Ro})^{2}}}-\epsilon \frac{1}{2 \mathrm{Ro}} h, \quad c_{2}=\sqrt{\frac{h}{\mathrm{Fr}^{2}}+\epsilon^{2} \frac{h^{2}}{(2 \mathrm{Ro})^{2}}}+\epsilon \frac{1}{2 \mathrm{Ro}} h .
$$

Les vitesses ouest-est $\left(c_{1}\right)$ et est-ouest $\left(c_{2}\right)$ ne sont alors plus identiques de par l'effet cosinus. On renvoie le lecteur à [8] pour de plus amples détails. On remarque que si $\epsilon$ est du même ordre que Ro, la perturbation apportée par l'effet cosinus est d'ordre un!

\section{Références}

[1] D. Bresch, B. Desjardins. Existence of global weak solutions for a 2D viscous shallow water equations and convergence to the quasi-geostrophique model, Comm. Math. Phys., 238 1-2 (2003) 211-223.

[2] D. Bresch, M. Gisclon, C.K. Lin. An example of low Mach (Froude) number effects for compressible flows with nonconstant density (height) limit, M2AN, 393 (2005) 477-486.

[3] D. Bresch, G. MÉtivier. Global existence and uniqueness for the lake equations with vanishing topography : elliptic estimates for degenerate equations. Nonlinearity, 193 (2006) 591-610.

[4] I. Gallagher, L. Saint-Raymond. Mathematical study of the betaplane model : Equatorial waves and convergence results. To appear in Mémoires de la Société Mathématique de France, (2007).

[5] J.-F. Gerbeau, B. Perthame. Derivation of viscous Saint-Venant system for laminar shallow water : Numerical validation. Discrete and Continuous Dynamical Systems, Serie B (1) (2001) 89-102.

[6] C. D. Levermore, M. Sammartino. A shallow water model with eddy viscosity for basins with varying bottom topography. Nonlinearity, 14 (2001) 1493-1515.

[7] P.-L. Lions. Mathematical topics in fluid mechanics, Vol.2, Compressible models. Clarendon Press, Oxford, (1998).

[8] C. Lucas. Cosine effect on Shallow Water equations and mathematical properties. En préparation, (2007).

[9] C. Kazantsev, C. Lucas, A. Rousseau. New Developments and Cosine Effect in the Viscous Shallow Water and Quasi-Geostrophic Equations. En préparation, (2007).

[10] F. MARChE. Derivation of a new two-dimensional viscous shallow water model with varying topography, bottom friction and capillary effects. European J. Mech. B / Fluids, (2006).

[11] J. PedLosky. Geophysical fluid dynamics. Springer-Verlag, New York, (1979).

[12] A. Oron, S.H. Davis, S.G. Bankoff. Long-Scale evolution of thin liquid films. Rev. Mod. Phys., 693 (1997) $931-981$.

[13] J.-P. VILA. Thin film models and Shallow Water equations. Communication privée, (2007). 